\title{
Kernos
}

Revue internationale et pluridisciplinaire de religion grecque antique

$13 \mid 2000$

Varia

Philippe BORGEAUd (avec Nicole DURISCH, Antje KOLDE et Grégoire SOMMER), La mythologie du matriarcat. L'atelier de Johann Jakob Bachofen

\section{Vinciane Pirenne-Delforge}

\section{OpenEdition \\ Journals}

\section{Édition électronique}

URL : http://journals.openedition.org/kernos/1351

DOI : 10.4000/kernos.1351

ISSN : 2034-7871

\section{Éditeur}

Centre international d'étude de la religion grecque antique

Édition imprimée

Date de publication : 1 janvier 2000

ISSN : 0776-3824

\section{Référence électronique}

Vinciane Pirenne-Delforge, «Philippe boRgeAud (avec Nicole dURISCH, Antje kolde et Grégoire sommer), La mythologie du matriarcat. L'atelier de Johann Jakob Bachofen », Kernos [En ligne], 13 | 2000, mis en ligne le 16 juin 2011, consulté le 22 septembre 2020. URL : http://journals.openedition.org/kernos/ 1351 ; DOI : https://doi.org/10.4000/kernos.1351 
trouve des antécédents de l'utilisation parodique de la gastronomie dans la poésie iambique et le drame satirique, mais Épicarme deviendra celui qui introduira des personnages gourmands, ivres ou cuisiniers. Dans toute la comédie athénienne, la nourriture est un motif humoristique, mais spécialement dans le "filone disimpegnato » de la comédie archaïque et dans la comédie postérieure. - Carmen Morenilla Talens ("El hetaîros en Menandro", p. 227-269) étudie le couple formé par le protagoniste et son ami qui l'aide, ce dernier parfois remplacé dans ses fonctions par un parasite ou un serviteur, dans un schéma dramatique qui aura un grand succès dans la suite. - Le travail du professeur Jesús Lens Tuero, récemment décédé, auquel le prof. López Férez dédie sa contribution, ("Comedia e Historiografía: Ctesias de Cnido", p. 271-306) porte sur les concommitances entre les procédés historiographiques de Ctésias de Cnide et ceux de la comédie, au sujet des endroits, des personnages, des structures narratives, des types fixés et quelque peu stéréotypés. - Pour conclure, il y a trois articles sur l'influence de ce genre dans la littérature postérieure: Andrés Pociña ("Menandro en la comedia latina", p. 345-367) observe l'influence de cet auteur à Rome; Antonio Bravo García ("El Aristófanes de las bibliotecas de la Comunidad de Madrid: una ojeada a los fondos de El Escorial", 369-386) fait une révision des fonds de l'Escorial en ce qui concerne Aristophane, du point de vue codicologique et paléographique, et l'éditeur du volume, Juan Antonio López Férez fait un "Estudio sobre la influencia de la comedia griega en la literatura española", (387455), nécessairement rapide mais avec une grande quantité de données, une source importante pour des études plus détaillées.

Angel Ruiz Pérez
(Universidad de Santiago de Compostela)

Philippe Borgeaud (avec Nicole Durisch, Antje Kolde et Grégoire Sommer), La mythologie du matriarcat. L'atelier de Johann Jakob Bachofen, Genève, Droz, 1999. 1 vol. $15 \times 22 \mathrm{~cm}, 252$ p. (Recherches et Rencontres. Publications de la Faculté des lettres de Genève, 13). ISBN : 2-600-00350-9.

Le mot de «matriarcat » fait immédiatement surgir tout un univers fantasmatique où les femmes ont le pouvoir et l'exercent. Certains mythes anciens et certaines enquêtes ethnologiques, qu'elles soient antiques ou du temps des Lumières, évoquent en effet une série de peuplades ayant connu une domination féminine, en plus de la simple filiation matrilinéaire. Ces récits ont profondément marqué Johann Jakob Bachofen, qui a publié sur le sujet, en 1861, un gros ouvrage intitulé Das Mutterrecht, Le Droit maternel (désormais disponible dans la traduction française d'Étienne Barilier, Lausanne, 1996). Ph. Borgeaud s'est penché, avec quelques collaborateurs, sur les archives de Bachofen conservées à Bâle, pour éclairer le cheminement souvent tortueux et la longue maturation qui l'ont conduit à élaborer ses théories et à devenir ainsi le fondateur de l'idée matriarcale, même si le mot comme tel ne se trouve pas dans l'œuvre. L'étude de $\mathrm{Ph}$. Borgeaud nous ouvre donc bien la porte de "l'atelier de J.J. Bachofen ». L'ouvrage fait tout d'abord le point sur la notion de " gynécocratie ». L'analyse du mot et de la chose en Grèce ancienne montre qu'il s'agit essentiellement d'une exploration imaginaire des limites du social, qui inverse les valeurs fondatrices de la communauté citoyenne. Mais c'est dans la perspective d'une véritable genèse historique, et pas seulement d'un renversement symbolique des valeurs, que Bachofen, après Lafitau mais sans lien de dépendance avec ce dernier, va progressivement penser le «droit maternel ». Par contre, la dette de Bachofen à l'égard des théories de son temps sur les notions de mythe et de symbole est assurée : Heyne, Creuzer, Gerhard, K.O. Müller, Preller forment l'horizon intellectuel sur 
lequel vient s'inscrire la réflexion de Bachofen. L'analyse minutieuse des archives bâloises est ensuite proposée avec une grande clarté, en dépit de la difficulté de tels documents manuscrits et, surtout, des entrelacs complexes, souvent ambigus, de la pensée de leur auteur. Née de l'intérêt de Bachofen pour l'ancienne Italie et d'un ouvrage inachevé sur le sujet, la théorie du matriarcat est un système qui fait de la gynécocratie une institution universelle, une étape nécessaire appartenant au plus lointain passé, avec le culte d'une terre-mère à l'origine de toutes les reli gions. Bachofen dessine les phases de l'évolution des humains vers la spiritualisation de l'espèce, liée à la prédominance progressive du père, tout en soulignant la fragilité de la victoire de l'esprit et l'ombre toujours menaçante des puissances féminines autrefois écartées. Quelques dossiers extraits par Ph. Borgeaud de cette masse de données illustrent les modes de raisonnements à l'œuvre dans cette vaste entreprise (Hypsipyle, Bellérophon, Massagètes/Scythes/Garamantes/Arabes, discours de Diotime chez Platon, Dionysos et Apollon). Le dernier chapitre évoque l'Égypte de Bachofen, celle dont l'intérêt lui est révélé par la lecture du De Iside et Osiride de Plutarque. Le Bâlois y trouve l'occasion de réfléchir sur les rapports entre masculin et féminin, et la préséance d'Isis sur Osiris devient un argument central de la thèse du caractère gynécocratique de la civilisation égyptienne qu'il développera dans le Mutterrecht.

Ce livre original et clair en dépit de la complexité de son sujet fournit au public francophone une clé de lecture extrêmement utile pour aborder à la fois la réflexion autour du mythe et du symbole dans l'Allemagne du xix ${ }^{e}$ siècle, et les méandres d'une œuvre particulièrement foisonnante, idéologiquement très marquée. Certains rameaux continuent pourtant de fleurir autour de telles idées historiquement contestables : ce n'est pas le moindre mérite de l'ouvrage coordonné par Ph. Borgeaud de nous inviter à les débusquer.

Vinciane Pirenne-Delforge (Université de Liège)

\section{Actes de colloques, ouvrages collectifs et mélanges}

Athanassiadi Polymnia, Frede Michael (éds), Pagan Monotheism in Late Antiquity, Oxford, Clarendon Press, 1999.

M.L. WeST, Towards monotheism, p. 21-40; M. Frede, Monotheism and pagan philosophy in later antiquity, p. 41-67; J. DinLon, Monotheism in the gnostic tradition, p. 69-79; St. Mrtchell, The cult of theos bypsistos between pagans, Jews, and Christians, p. 81-148; P. Athanassiad, The Chaldean oracles: theology and theurgy, p. 149-183; W. Liebeschuetz, The significance of the Speech of Praetextatus, p. 185-205.

Bats M., D'Agostino B. (éds), Euboica. L'Eubea e la presenza euboica in Calcidica e in Occidente. Atti del Convegno Internazionale di Napoli, 13-16 novembre 1996, Napoli, 1998 (Coll. du Centre Jean Bérard, 16 / AION(ArchStAnt), Quaderno 12).

Irad MALKIN, Ithaka, Odysseus and the Euboeans in the eightb century, p. 1-10; Albio Cesare CAssio, La cultura euboica e lo suiluppo dell'epica greca, p. 11-22; François DE Polignac, Navigations et fondations: Héra et les Eubéens de l'Égée à l'Occident, p. 23-29; Denis Knoepfler, Le béros Narkittos et le système tribal d'Érétrie, p. 105-108; Sandrine HuBER, Érétrie et la Méditerranée à la lumière des trouvailles provenant d'une aire sacrificielle au Nord du Sanctuaire d'Apollon Daphnéphoros, p. 109-133; Béatrice BLANDIN, Recherches sur les tombes à inbumation de l'Hérôon d'Érétrie, p. 135-146; Claude BÉRARD, 